\title{
Energy radiation of moving cracks
}

\author{
S. Fratini ${ }^{1}$, O. Pla ${ }^{1}$, P. González ${ }^{2}$, F. Guinea ${ }^{1}$ and E. Louis ${ }^{3}$ \\ 1 Instituto de Ciencia de Materiales, Consejo Superior de Investigaciones Científicas, Cantoblanco, E-28049 Madrid, Spain. \\ 2 Departamento de Física. Universidad Carlos III. Butarque 15. Leganés. 28913 Madrid. Spain. \\ 3 Departamento de Física Aplicada, Universidad de Alicante, Apartado 99, E-03080 Alicante, Spain.
}

(November 1, 2018)

\begin{abstract}
The energy radiated by moving cracks in a discrete background is analyzed. The energy flow through a given surface is expressed in terms of a generalized Poynting vector. The velocity of the crack is determined by the radiation by the crack tip. The radiation becomes more isotropic as the crack velocity approaches the instability threshold.
\end{abstract}

\section{INTRODUCTION}

The dynamics of cracks in brittle materials are being extensively studied [1,2], and a wealth of instabilities and patterns have been observed as a function of control parameters such as the applied strain [3 7], or thermal gradients [8]. The theoretical analysis of moving cracks was initiated long ago [9 11, with the study of exact solutions for cracks moving at constant velocity. These studies have been extended to a variety of different situations [12,13. Alternatively, analytical approximations to the leading instabilities of a moving tip have been proposed [14.

The simplest discrete model which captures the main features of cracks in brittle materials is a a lattice with central forces (springs) between nearest neighbors, whose bonds lose the restoring force above a given threshold [15] (for extensions see also [16]). This model, or simplifications of it which leave out the vectorial nature of the strain field, has been extensively used in modelling moving cracks 2 2, 13, 17, 19, although models which deal with the microscopic structure of the system are also being considered 20 23]. Alternatively, various continuum models, which describe the fractured zone in terms of additional fields, have been proposed [24,25].

Discrete and continuum models of cracks differ in a variety of features. It is known that the discrete models used so far cannot describe a fracture zone at scales other than the size of the lattice cell in the calculations [18,26, although, even for a canonical material such as PMMA, the fracture zone has a dimension much larger than the size of its molecular building blocks 27.

Another important difference between discrete and continuum models is the existence of radiation from the tip of the moving crack, due to the existence of periodic modulations in the velocity in the presence of an underlying lattice. In this sense, a lattice model for cracks is the simplest example where radiation due to the scattering of elastic waves by deviations from perfect homogeneity can be studied. These processes have been observed in experiments [6,28 30], and it has been argued that they are responsabible for some of the crack instabilities [30].
In the present work, we study the energy radiated by a crack moving at constant velocity in a discrete lattice. We use a generalization of the scheme discussed in $[$ i. The general method used is explained next. Section III presents the main features of the results. The physical implications of the results is discussed in section IV.

The problem of sound emission by moving cracks has been addressed, within a different scheme, by [31]. Insofar as the two approaches can be compared, the results are compatible. Finally, radiation of moving cracks along the edge of the crack can be important in understanding the roughness of the crack surface [32]. We will focus on the radiation along the crack surface, and into the bulk of the sample. Experiments [30] and simulations [2] suggest that this type of radiation can play a role in the observed instabilities of the crack tip.

\section{THE METHOD}

We study discrete models of elastic lattices in two dimensional stripes, as discussed in 17,18]. The underlying lattice is hexagonal, with nearest neighbor forces. Bonds break when their elongation exceed a given threshold, $u_{t h}$, and under a constant strain at the edges, which, scaled to the width of the stripe, we write as $u_{0}$. We study models with and without dissipation in the dynamics of the nodes. Results depend on the ratio $u_{t h} / u_{0}$ (see section III for further details).

\section{A. Energy considerations}

In the absence of dissipation, the total kinetic plus elastic energy must be conserved. In a continuum model, in the absence of radiation, energy conservation leads to a global and to a local constraint, for cracks moving at constant speed, $v$ :

i) In the absence of radiation, the region well behind the crack tip has relaxed to equilibrium, while the region ahead of it is under the applied strain. The relaxed region grows at the expense of the region under strain, 
at constant rate $\propto u_{0}^{2} v W$ where $W$ is the width of the stripe [18]. Energy is transferred to the crack at this rate. As the energy stored in the crack grows at rate $\propto u_{t h}^{2} v$, the crack can only propagate (without radiation) for a fixed value of $u_{t h} / u_{0}$. Note that continuum solutions for radiationless cracks moving at constant speed [9] do not specify a parameter equivalent to $u_{t h}$, so that they do not conflict with energy conservation.

ii) The only position at which elastic energy is used to increase the size of the crack is the crack tip. Thus, at the crack tip the flux of elastic energy should be equal to the energy invested in enlarging the crack [1,33. In continuum models, this local constraint leads to an equation of the type:

$$
\Gamma=A(v) G
$$

where $\Gamma$ is the crack energy per unit length $\left(\Gamma \propto u_{t h}\right.$ in our lattice model), $G$ is proportional to the stress intensity factor at the crack tip $\left(G \propto u_{0}^{2}\right)$, and $A(v)$ is a universal function which goes from 1 at $v=0$ to 0 at $v=v_{R}$ where $v_{R}$ is the Rayleigh speed.

In lattice models, the energy arguments have to be modified because of the presence of radiation of elastic waves. If we assume that the difference between continuum and lattice models is small, we can use the perturbative scheme discussed in 34 38. The crack tip velocity undergoes oscillations at frequency $\omega=v / a$, where $a$ is the lattice constant, and amplitude $f$. In order to estimate the energy radiated from the tip, we have to extend the perturbative expansion to second order. We will not attempt here to calculate this expansion rigorously. However, from the knowledge of the leading term [36, 38, we can infer that the radiation due to a perturbation of frequency $\omega$ should go as $B \omega^{2}$, where $B$ is a positive constant. The global constraint i) implies that $f^{2}(v / l)^{2} \propto 1-k\left(u_{t h} / u_{0}\right)^{2}$, where $l$ is the crack length and $k$ is a constant. The radiation also has to be taken into account when balancing the energy absorbed at the crack tip, ii) above. The corrections to eq. (1) lead to a condition of the type:

$$
\frac{v}{v_{R}}=\mathcal{A}\left[\frac{\left(\frac{u_{t h}}{u_{0}}\right)^{2}}{c+c^{\prime}\left(1-k\left(\frac{u_{t h}}{u_{0}}\right)^{2}\right)}\right]
$$

where $c$ and $c^{\prime}$ are constants, and $\mathcal{A}$ is proportional to the inverse of the function $A$ defined in eq.(1), the magnitude in brackets being its argument. Thus, the existence of radiation in discrete models allows for the existence of a continuum of solutions $v\left(u_{0} / u_{t h}\right)$ for a given range of $u_{0} / u_{t h} 12,13,17,19$. Note that the existence of solutions which do not violate energy conservation does not imply that these solutions are stable. Full dynamical simulations of lattice models 17 19 suggest that inertial cracks (without dissipation) accelerate until they reach speeds comparable to those predicted by the Yoffe criterion [9], and then bifurcate.

\section{B. Energy flux: continuum elasticity}

In the following, we will reformulate the concepts discussed in [1] in order to make them more amenable for extensions to lattice models, dicussed in the next subsection.

39:

$$
\begin{aligned}
\mathcal{H} & =\mathcal{H}_{\text {kin }}+\mathcal{H}_{\text {elastic }} \\
\mathcal{H}_{\text {kin }} & =\int d^{D} r \frac{\rho}{2}\left(\frac{\partial \overrightarrow{\mathbf{u}}(\overrightarrow{\mathbf{r}})}{\partial t}\right)^{2} \\
\mathcal{H}_{\text {elastic }} & =\int d^{D} r \frac{\lambda}{2}\left(\sum_{i} u_{i i}\right)^{2}+\mu \int d^{D} r \sum_{i j} u_{i j}^{2}
\end{aligned}
$$

where $D$ is the spatial dimension, $\rho$ is the mass density, $\lambda$ and $\mu$ are Lamé coefficients, $\overrightarrow{\mathbf{u}}(\overrightarrow{\mathbf{r}})$ denotes the displacements at position $\overrightarrow{\mathbf{r}}$, and the $u_{i j}$ 's define the strain tensor:

$$
u_{i j}(\overrightarrow{\mathbf{r}})=\frac{1}{2}\left(\frac{\partial u_{i}}{\partial r_{j}}+\frac{\partial u_{j}}{\partial r_{i}}\right)
$$

The equations of motion satisfied by $\overrightarrow{\mathbf{u}}(\overrightarrow{\mathbf{r}})$ can be written as:

$$
\rho \frac{\partial^{2} u_{i}}{\partial t^{2}}=-\sum_{j} \frac{\partial}{\partial r_{j}} \sigma_{j i}
$$

where $\sigma_{j i}=\partial \mathcal{H}_{\text {elastic }} / \partial u_{i j}$ is the stress tensor.

The time derivative of the total energy $E_{\Omega}$ within a region $\Omega$ is:

$$
\begin{aligned}
\frac{\partial E_{\Omega}}{\partial t} & =\frac{\partial}{\partial t} \int_{\Omega} d^{D} r\left[\mathcal{H}_{\text {kin }}+\mathcal{H}_{\text {elastic }}\right] \\
& =\int_{\Omega} d^{D} r\left[\rho \frac{\partial \overrightarrow{\mathbf{u}}}{\partial t} \frac{\partial^{2} \overrightarrow{\mathbf{u}}}{\partial t^{2}}+\frac{\partial u_{i j}}{\partial t} \sigma_{i j}\right] \\
& =-\int_{\Omega} d^{D} r \frac{\partial}{\partial r_{j}}\left(\sigma_{i j} \frac{\partial u_{i}}{\partial t}\right)
\end{aligned}
$$

so that the vector $\overrightarrow{\mathbf{P}}(\overrightarrow{\mathbf{r}})$ with components $P_{j}=$ $\sum_{i} \sigma_{i j} \partial u_{i} / \partial t$ plays the same role as the Poynting vector in electrodynamics. The energy flux through an element of area $d \overrightarrow{\mathbf{S}}$ is given by $\overrightarrow{\mathbf{P}} d \overrightarrow{\mathbf{S}}$. Note, however, that, unlike in electromagnetism, the equations of elasticity have not Lorentz invariance (there are two sound velocities), and it is not possible to define a four vector combining $\overrightarrow{\mathbf{P}}$ and the energy density. The energy transferred to the outside of this region remains defined as the flux of the vector $\overrightarrow{\mathbf{P}}$ through the surface bounding $\Omega$. In the presence of dissipation, we still use $\overrightarrow{\mathbf{P}}$ as defined in Eq. (6) in the understanding that what viscosity does is to trigger the partial absorbtion of the radiated enegy whithout 
changing the direction in which it is emitted. The vector $\overrightarrow{\mathbf{P}}$ will be our starting point in the study of the energy flux of a moving crack.

\section{Energy flux: lattice model}

We will compute numerically the radiation of energy in a discrete model, defined as a hexagonal two dimensional lattice with nearest neighbor forces [15,17, 18. The energy is given by the sum of a kinetic term, associated to the velocities of the nodes, and an elastic term, due to the deformation of the bonds. The variation of the elastic energy of a given bond with time can be written as:

$$
\frac{\partial E_{i j}}{\partial t}=k\left[\left(\overrightarrow{\mathbf{u}}_{i}-\overrightarrow{\mathbf{u}}_{j}\right) \overrightarrow{\mathbf{n}}_{i j}\right] \frac{\partial\left[\left(\overrightarrow{\mathbf{u}}_{i}-\overrightarrow{\mathbf{u}}_{j}\right) \overrightarrow{\mathbf{n}}_{i j}\right]}{\partial t}
$$

where $k$ is the force constant, and $\overrightarrow{\mathbf{n}}_{i j}$ is a unit vector in the direction of the bond. We distribute this energy among the two nodes connected by the bond, so that we can write the total elastic energy within a given region as a sum of the contributions of the nodes within that region. The variation in the kinetic energy at node $i$ is:

$$
\frac{\partial K_{i}}{\partial t}=-k \sum_{j} \frac{\partial\left(\overrightarrow{\mathbf{u}}_{i} \overrightarrow{\mathbf{n}}_{i j}\right)}{\partial t}\left[\left(\overrightarrow{\mathbf{u}}_{i}-\overrightarrow{\mathbf{u}}_{j}\right) \overrightarrow{\mathbf{n}}_{i j}\right]
$$

The variation of the total energy within a given region is calculated by summing over all bonds within that region. The kinetic and elastic contributions for all bonds outside the edge of the region cancel. We are left with surface terms only, as in the continuum model described earlier. The surface contributions can be written as a sum of terms associated to the bonds which connect a node within the region under study and a node outside. Thus, a surface which includes a given node and has a given orientation leads to an energy flux across it which can be calculated from a weighted sum of the positions and velocities of the bonds which connect that node to its neighbors. As we can associate to each surface orientation an energy flux, we can define the lattice Poynting vector, in analogy to the analysis done for the continuum model. We will use this discrete Poynting vector in the discussion of the energy dissipation of a moving crack below.

\section{RESULTS}

The discrete equations of motion in a two dimensional lattice of a given size are integrated numerically as discussed in detail elsewhere [18]. The lattice is maintained under constant load at the edges. In order to obtain cracks moving at constant velocities, a notch is induced at one side, which is gradually enlarged, along a straight line, until the stress buildup leads to the spontaneous propagation of the crack. The crack position, as function of time, is shown in Figure 1, for two different applied strains. The calculations show that the crack propagates freely at a constant velocity in the steady state. Our method for the calculation of the properties of cracks moving at constant speeds should lead to the same results as other techniques.

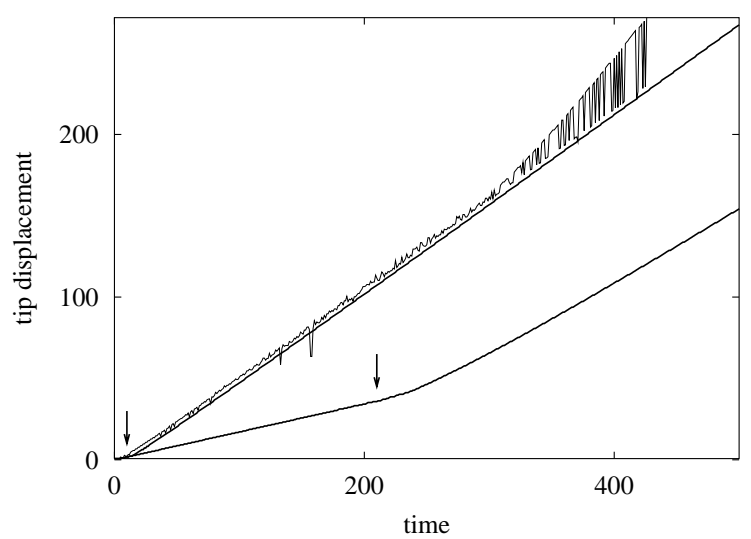

FIG. 1. Crack tip displacement versus time for cracks under two different applied strains (upper thick line: $u_{0}=0.08$; lower thick line: $u_{0}=0.02$ ) and zero viscosity. The arrow indicates the position of the notch beyond which the stresses at the crack tip exceed the threshold stress, and the crack propagates freely. The thin line is for $u_{0}=0.08$, but allowing for the branching instability.

Instabilities are avoided by allowing only the bonds directly ahead of the crack to break. In other words, we force the crack to propagate straightly (with no branching). The simulations are performed in systems of $400 \times 120$ lattice sites, where we have checked that finite size effects on the steady state velocity are less than 1 percent.

\section{A. Crack velocity}

Figure 2 shows the steady state velocity $v$ as a function of the applied strain $u_{0}$, for two different values of the viscosity ( $\eta=0$ and $\eta=0.8$ in our units). The crack velocity increases monotonically with $u_{0}$ and asymptotically tends to its limiting value $c_{R}=0.571$, the Rayleigh velocity in units where the force constant $k=1$ and the mass per site $m=1$ [16]. Due to lattice trapping, there is a minimum allowed $u_{0}$ whose value is roughly independent of $\eta[13,40$, which in turn leads to a minimum crack speed which depends strongly on $\eta$. The arrow marks the instability that would occur if the crack were not constrained to move on a straight line. 


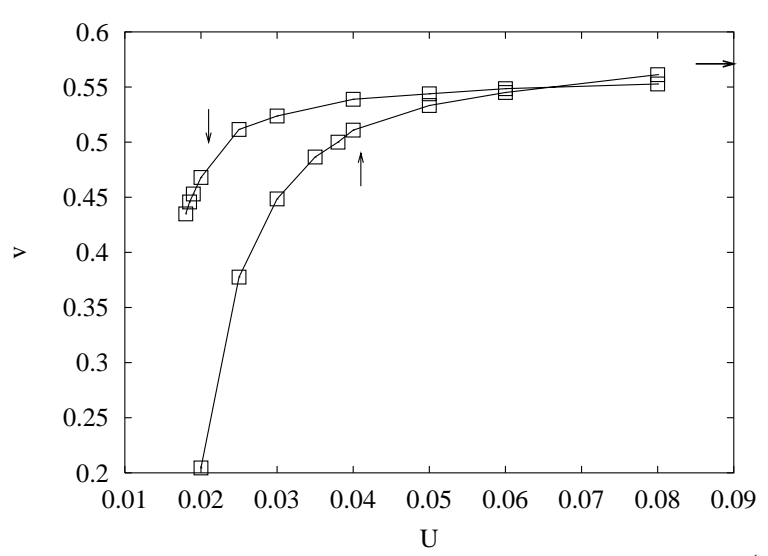

FIG. 2. Crack velocity versus external strain, for $\eta=0$ (upper curve) and $\eta=0.8$ (lower curve). The arrow on the right indicates the Rayleigh velocity $v_{R}$. The vertical arrows mark the (avoided) branching instability (see text). The threshold for breaking is $u_{t h}=0.1$

\section{B. Elastic energy and hoop stress}
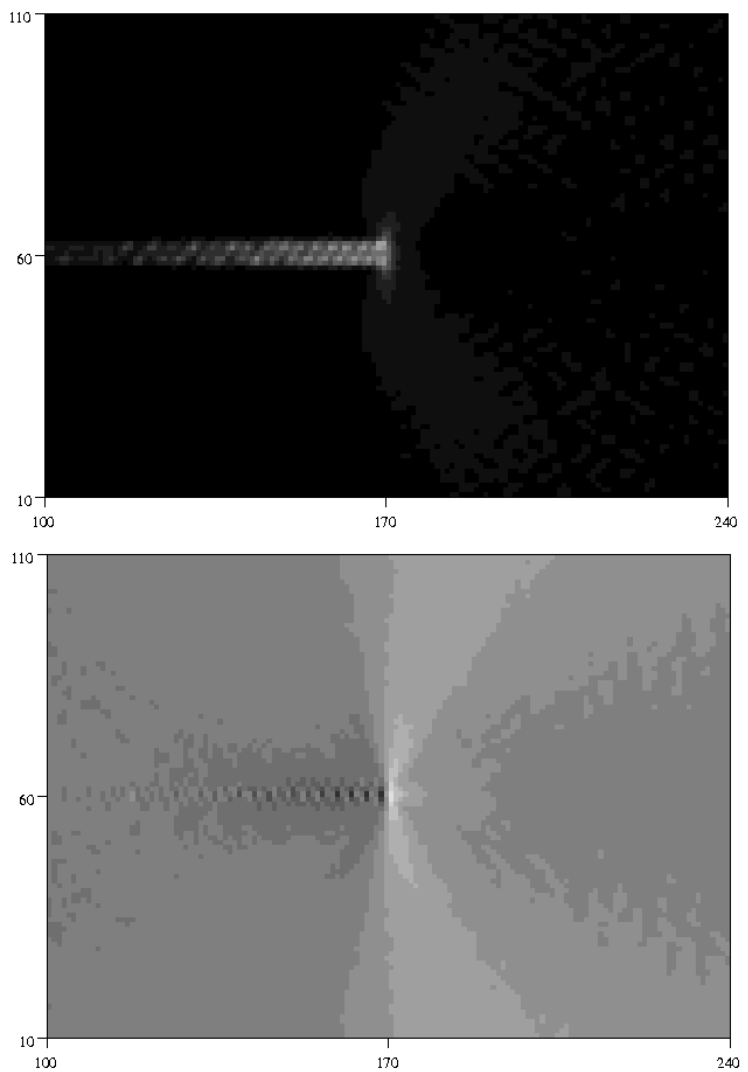

FIG. 3. Density of elastic energy (upper panel) and hoop stress (lower panel) for an inertial crack $(\eta=0)$ moving under an applied strain $u_{0}=0.02$, below the branching instability. The crack moves from left to right, and the tip is located at a point of coordinates 170,60 .
Figure 3 shows snapshots of the density of elastic energy and the hoop stress at a given time $t_{0}$, for a steady inertial crack $(\eta=0)$ moving at a velocity below the branching threshold (the applied strain is $u_{0}=0.02$, cf. figure 2).

The density of elastic energy (Fig.3, upper panel) has a sharp peak at the crack tip. In the near region (a few lattice spacings away from the tip), we see that the distribution of elastic energy is very anisotropic: it is sizeable in the direction perpendicular to the crack motion, where it decays smoothly with the distance, and all along the crack, where it has an oscillating behavior. This behavior is reminiscent of the Rayleigh waves which propagate on the crack surface (see section III below). At larger distances (of the order of the linear dimensions of the system), the elastic energy is smoother and has a broad maximum ahead of the tip, around a given angle of the order of $\theta \approx \pi / 3$ from the crack direction. We cannot be conclusive about this maximum being intrinsic in nature, or rather being related to the symmetry of the underlying triangular lattice (see [19] for a more detailed discussion of this point).

The hoop stress (Fig. 3, lower panel) shows a very similar behavior, with strong oscillations all along the crack, and maxima perpendicular to the crack motion, the maximum shifting from $\theta \approx \pi / 2$ to $\theta \approx \pi / 3$ with increasing distance from the tip.

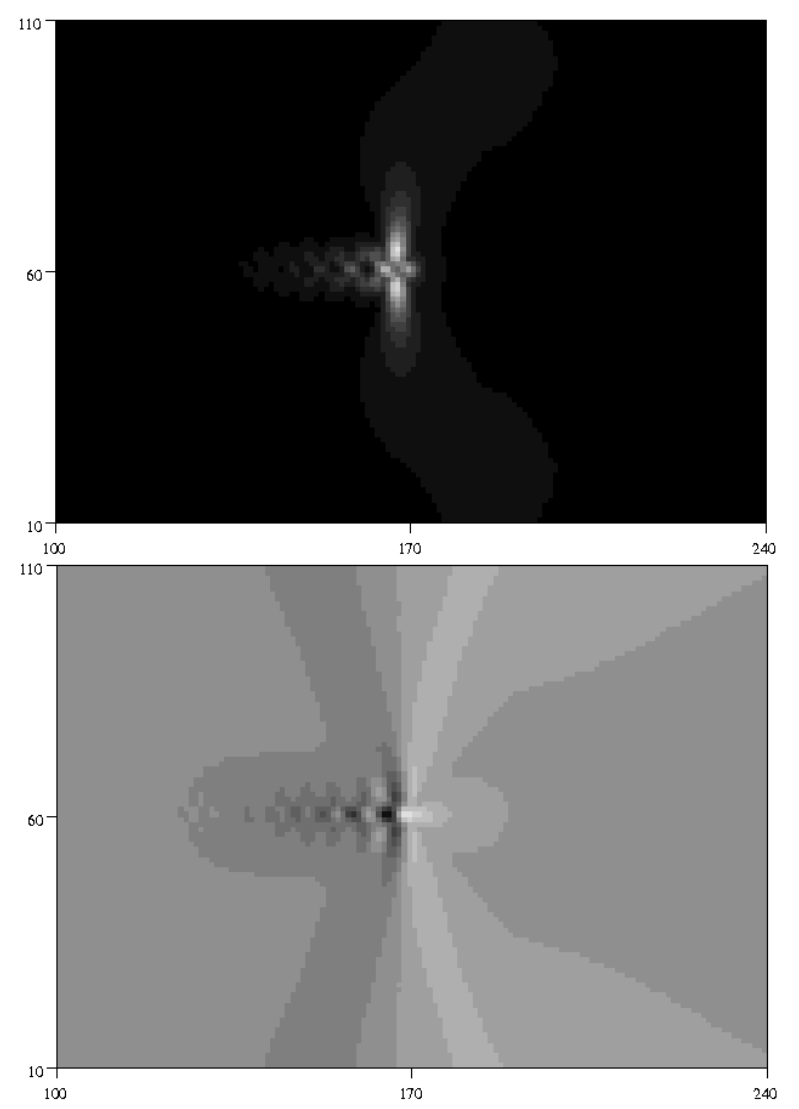


FIG. 4. Density of elastic energy (upper panel) and hoop stress (lower panel) for an inertial crack $(\eta=0)$ moving under an applied strain $u_{0}=0.08$, well above the branching instability.

Figure 1 is the same as Fig. 3, but for a crack moving at a velocity well above the branching threshold (the applied strain is $\left.u_{0}=0.08\right)$. We notice that the distribution of elastic energy and hoop stress has changed qualitatively: the bulk features in the direction perpendicular to the crack motion now dominate over the oscillating part along the crack. The latter decay more rapidly and eventually disappear far behind the tip.
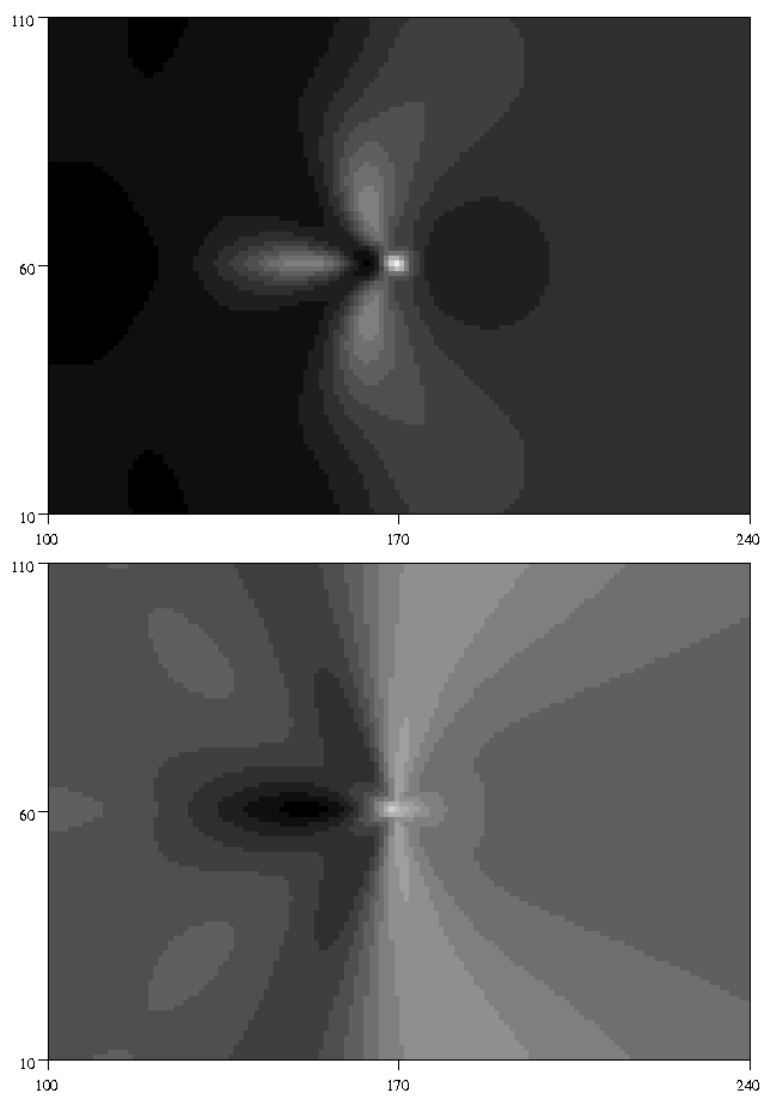

FIG. 5. Density of elastic energy (upper panel) and hoop stress (lower panel) for a dissipative crack $(\eta=0.8)$ moving under an applied strain $u_{0}=0.08$.

The elastic energy and hoop stress corresponding to a dissipative crack $\left(\eta=0.8, u_{0}=0.08\right)$ are shown in fig. 5. Although the overall characteristics are similar to the inertial case, with maxima at the tip and in the direction transverse to the crack, the distribution of stresses is much smoother. Moreover, the oscillations associated with Rayleigh waves along the crack are washed out by viscosity, being replaced by a single broad maximum behind the tip.

\section{Radiation}
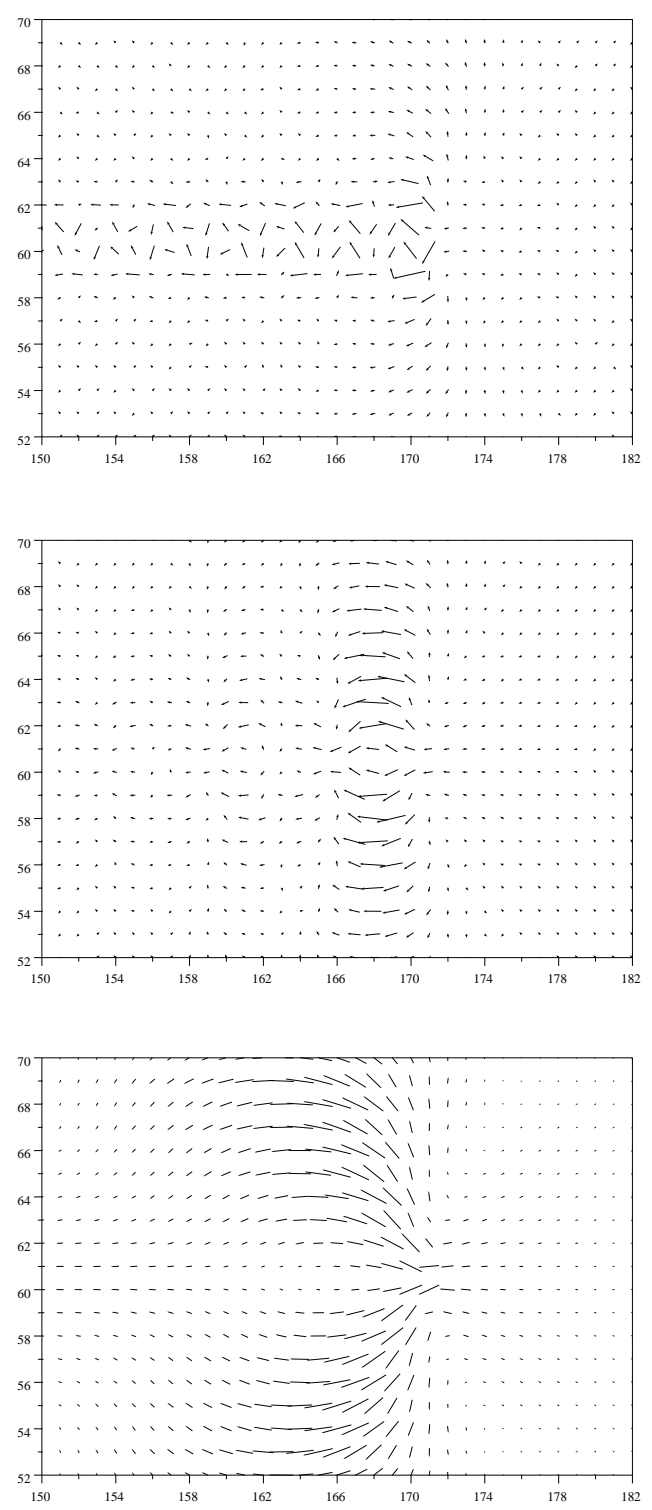

FIG. 6. Poynting vector field representing the radiation propagating in the vicinity of the crack tip. Upper panel: slow inertial crack (same parameters as fig. 3); center panel: fast inertial crack (same as fig. (4)); lower panel: dissipative crack (same as fig. 1 ).

The above results can be better understood by analyzing the Poynting vector field, which represents the flux of energy being radiated at a given point in the system. As was stated in the introduction, emission of sound waves is expected since the crack tip moves in a discrete medium, therefore acting as a source of radiation at a frequency $\omega=v / a$, the ratio of the crack speed to the lattice spac- 
ing. Moreover, one expects a net flux of energy in the direction opposite to the crack motion, corresponding to the elastic energy released from the region ahead of the tip, which allows the crack to move.

As can be seen in the first panel of figure 6, at such moderate crack speeds most of the energy is radiated in the form of Rayleigh waves propagating backwards along the crack, with a wavelength comparable with (but not equal to) the lattice spacing $a$. Despite the fact that $\eta=0$, such waves are seen to decay at long distances behind the tip (they decay into bulk waves, the oscillating bonds on the crack surface acting themselves as sources of radiation). In addition, there is also a weaker emission of bulk waves from the tip, responsible for the observed maximum in the direction perpendicular to the crack motion.

At high crack speeds, on the other hand (cf. center panel in fig. 6), it is the bulk radiation which dominates the emission pattern. Moreover, shadow images of the near-field appear behind the tip (the strongest one being at around $x=161$ ).

In the case of viscous cracks (lower panel in figure 6), the emission pattern is entirely dominated by bulk waves, and Rayleigh oscillations disappear in agreement with the reults of fig. 5 .

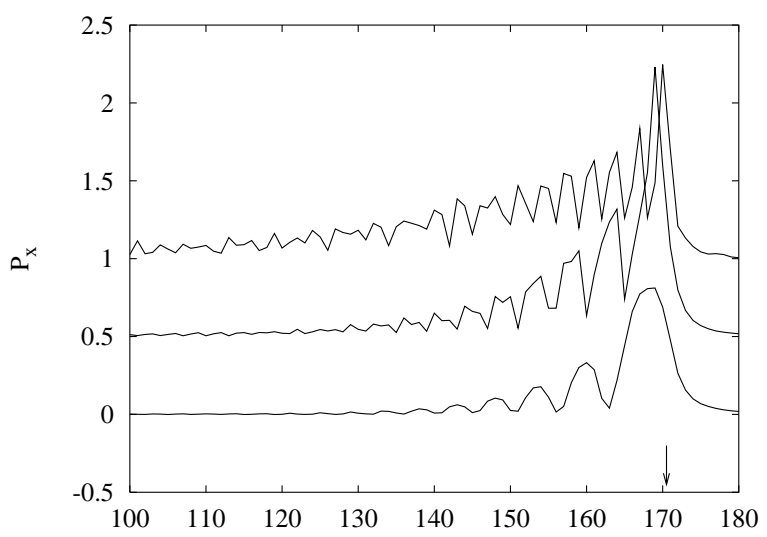

FIG. 7. The component of the Poynting vector along an inertial crack, as a function of the coordinate $x$, normalized to the total energy flowing through the sample, $\propto u_{0}^{2} v W$ (see text). From top to bottom: $u_{0}=0.02,0.04,0.08$. The vertical arrow marks the tip position. The curves are shifted by a vertical offset for clarity purposes.

In order to analyze the behavior of Rayleigh waves along inertial cracks, we plot in figure 7 the component of the Poynting vector $P_{x}$ parallel to the crack direction, at the surface of the crack, for different values of the applied strain. The data are normalized to the difference in mechanical energy of a line far ahead from the crack, and a line far behind (this energy, which scales as $u_{0}^{2} v W$, is transferred to the crack in the fracture process). The figure clearly shows that the wavelength of surface waves as well as their decay rate increase with the crack velocity.

\section{CONCLUSIONS}

We have analyzed the nature and influence of radiation in the propagation of cracks in discrete systems. For the lattice and force models that we have studied, we find:

i) Cracks in lattice models radiate energy, even when the average velocity is constant and they move along a straight line. This can be understood by assuming that the crack tip undergoes oscillations at frequencies $n v / a$, where $v$ is the velocity of the crack and $a$ is the lattice constant.

ii) Radiation allows for the existence of a continuum of solutions of moving cracks at constant velocity. The balance of static elastic and crack energy is compensated by the radiation from the crack tip.

iii) At low velocities, most of the radiation is in Rayleigh waves along the surface of the crack. At velocities comparable to the Rayleigh velocity, a significant fraction of the radiated energy is in bulk waves with a more isotropic distribution.

iv) Viscosity allows for a faster exchange of the elastic energy stored ahead of the crack tip into other forms of energy. This can help to explain the increased stability of straight cracks in the presence of viscosity.

Among the questions which remain unsolved is the relation of the radiation to the instabilities of the crack tip. Our results suggest that inertial cracks accelerate along a straight line, until they attain speeds compatible with Yoffe's criterion [9]. On the other hand, the radiation of the crack tip becomes more isotropic at high velocities. It is unclear whether the continuum approach suffices to understand the instability observed in dynamical simulations of discrete models, or if the radiation from the tip of the crack plays a role in the instability. Note that the calculated instability occurs at higher velocities than the instabilities observed experimentally.

\section{ACKNOWLEDGEMENTS}

We are thankful to R. Ball, P. Español, M. Marder, T. Martín, A. Parisi, M. A. Rubio and I. Zúñiga for helpful discussions. Financial support from grants PB96-0875 and PB96-0085 (MEC, Spain), and FMRXCT980183 (European Union).

[1] L. B. Freund, Dynamic Fracture Mechanics (Cambridge University Press, New York, 1990). 
[2] J. Fineberg and M. Marder, Phys. Rep. 313, 1 (1999).

[3] J. Fineberg, S. P. Gross, M. Marder, and H. L. Swinney Phys. Rev. B 45,5146 (1992).

[4] J.F. Boudet, S. Ciliberto, and V. Steinberg, Europhys. Lett. 30, 337 (1995).

[5] J. F. Boudet, S. Ciliberto and V. Steinberg, J. Phys. II 6, 1493 (1996).

[6] E. Sharon, J. Fineberg and S. P. Gross, Phys. Rev. Lett. 74, 5096 (1995).

[7] E. Sharon, S.P. Gross, and J. Fineberg, Phys. Rev. Lett. 76, 2117 (1996).

[8] A. Yuse and M. Sano, Nature 362, 329 (1993).

[9] E.H. Yoffe, Phil. Mag. 42, 739 (1951).

[10] L. I. Slepyan, Doklady Sov. Phys. 26, 538 (1981); Doklady Sov. Phys. 37, 259 (1992).

[11] Sh. A. Kulamekhtova, V. A. Saraikin and L. I. Slepyan, Mech. Solids 19, 101 (1984).

[12] M. Marder and S.P. Gross, J. Mech. Phys. Solids 43, 1 (1995).

[13] D. A. Kessler and H. Levine, Phys. Rev. E 59, 5154 (1999); D. A. Kessler, Phys. Rev. E 61, 2348 (2000); D. A. Kessler and H. Levine, Phys. Rev. E 63, 16118 (2001).

[14] M. Adda-Bedia and M. Ben Amar, Phys. Rev. Lett. 76, 1497 (1996). M. Adda-Bedia, R. Arias, M. Ben-Amar and F. Lund, Phys. Rev. Lett. 82, 2314 (1999).

[15] E. Louis and F. Guinea, Europhys. Lett. 3, 871 (1987).

[16] P. Meakin, G. Li, L.M. Sander, E. Louis, and F. Guinea, J. Phys. A 22, 1393 (1989); O. Pla, F. Guinea, E. Louis, G. Li, L. M. Sander, H. Yan, and P. Meakin, Phys. Rev. A 42, 3670 (1990).

[17] O. Pla, F. Guinea, E. Louis, S. V. Ghaisas and L. M. Sander, Phys. Rev. B, 57, R13981 (1998).

[18] O. Pla, F. Guinea, E. Louis, S. V. Ghaisas y L. M. Sander, Phys. Rev. B 61, 11472 (2000).

[19] T. Martín, P. Español, M. A. Rubio, and I. Zúñiga, Phys. Rev. E 61, 6120 (2000).

[20] F. F. Abraham, Phys. Rev. Lett. 77, 869 (1996).

[21] S. J. Zhou, D. M. Beazley, P. S. Lomdahl, and B. L. Holian, Phys. Rev. Lett. 78, 479 (1997).

[22] R. K. Kalia, A. Nakano, A. Omeltchenko, K. Tsuruta, and P. Vashishta, Phys. Rev. Lett. 78, 2144 (1997).

[23] J. A. Hauch, D. Holland, M. P. Marder, and H. L. Swinney, Phys. Rev. Lett. 82, 3823 (1999).

[24] I. S. Aranson, V. A. Kalatsky, V. M. Vinokur, Phys. Rev. Lett. 85, 118 (2000).

[25] A. Karma, D. A. Kessler and H. Levine, Phys. Rev. Lett. 87, 045501 (2001).

[26] Z. P. Bazant and J. Planas, Fracture and Size Effect in Concrete and Other Quasibrittle Materials, CRC Press (New York), 1998.

[27] F. J. Gómez Sánchez, Ph. D. thesis. Universidad Politécnica de Madrid (1998).

[28] J. F. Boudet and S. Ciliberto, Phys. Rev. Lett. 80, 341 (1998).

[29] J. F. Boudet and S. Ciliberto, Physica D 142, 317 (2000).

[30] E. Sharon, G. Cohen and J. Fineberg, Nature 410, 68 (2001).

[31] R. C. Ball and A. Parisi, in preparation.

[32] E. Bouchaud, J.-P. Bouchaud, D.S. Fisher, S. Ramananthan, and J.R. Rice, preprint cond-mat/0108261.
[33] L. B. Freund, J. Mech. Phys. Sol. 20, 129 (1972); ibid 20, 141 (1972).

[34] J. R. Willis and A. B. Movchan, J. Mech. Phys. Solids, 43, 319 (1995).

[35] H. Larralde and R. C. Ball, Europhys. Lett. 30, 287 (1995).

[36] S. Ramanathan and D. S. Fisher, Phys. Rev. Lett. 79, 877 (1997).

[37] J. M. Morrissey and J. R. Rice, J. Mech. Phys. Solids, 46, 467 (1998).

[38] J. M. Morrissey and J. R. Rice, J. Mech. Phys. Solids, 48, 1229 (2000).

[39] L. Landau and E. Lifshitz, Theory of Elasticity, AddisonWesley, 1959.

[40] D. A. Kessler and H. Levine, Phys. Rev. E 60, 7569 (1999). 